\title{
DOUBLE SUMMABILITY FACTOR THEOREMS AND APPLICATIONS
}

\author{
EKREM SAVAŞ AND B. E. RHOADES
}

Abstract. We obtain sufficient conditions for the series $\sum \sum a_{m n}$, which is absolutely summable of order $k$ by a double triangular matrix method $A$, to be such that $\sum \sum a_{m n} \lambda_{m n}$ is absolutely summable of order $k$ by a double triangular matrix $B$. As corollaries we obtain a number of inclusion theorems.

Mathematics subject classification (2000): 40G99, 40G05, 40D15. factor.

Key words and phrases: absolute summability, weighted mean matrix, Cesáro matrix, summability

\section{REFERENCES}

[1] B. E. RHOADES, Inclusion theorems for absolute matrix summability methods , J. Math. Anal. Appl., 238, (1999), 82-90.

[2] B. E. RHOADES, On absolute normal double matrix summability methods, Glasnik Mat., 38, (2003), 57-73.

[3] B. E. RHOADES, EKREM SAVAŞ, General inclusion theorems for absolute summability of order $k \geqslant 1$, Math. Inequal. Appl., 8 () (2005), 505-520.

[4] B. E. RHOADES, EKREM SAVAŞ, General summability factor theorems and applications, Sarajevo J. Math., 13, (2005), 59-73. 